\title{
Stochastic 3D Modeling of Three-Phase Microstructures for Predicting Transport Properties: A Case Study
}

\author{
M. Neumann • B. Abdallah • L. Holzer • \\ F. Willot • V. Schmidt
}

Received: June 18, 2018 / Accepted: January 17, 2019

\begin{abstract}
We compare two conceptually different stochastic microstructure models, i.e., a graph-based model and a pluri-Gaussian model, that have been introduced to model the transport properties of three-phase microstructures occurring, e.g., in solid oxide fuel cell electrodes. Besides comparing both models, we present new results regarding the relationship between model parameters and certain microstructure characteristics. In particular, an analytical expression is obtained for the expected length of triple phase boundary per unit volume in the pluri-Gaussian model. As a case study, we consider 3D image data which show a representative cutout of a solid oxide fuel cell anode obtained by FIB-SEM tomography. The two models are fitted to image data and compared in terms of morphological characteristics (like mean geodesic tortuosity and constrictivity) as well as in terms of effective transport properties. The Stokes flow in the pore phase and effective conductivities in the solid phases are computed numerically for realizations of the two models as well as for the 3D image data using Fourier methods. The local and effective physical responses of the model realizations are compared to those obtained from 3D image data. Finally, we assess the accuracy of the two methods to predict permeability as well as electronic and ionic conductivities of the anode.
\end{abstract}

Keywords Stochastic microstructure modeling • effective conductivity • permeability $\cdot$ solid oxide fuel cells $\cdot 3 \mathrm{D}$ image data

\footnotetext{
M. Neumann · V. Schmidt

Institute of Stochastics, Ulm University, Helmholtzstraße 18, 89069 Ulm, Germany

Tel.: +49731-50-23617

E-mail: matthias.neumann@uni-ulm.de

B. Abdallah · F. Willot

MINES ParisTech, Centre for Mathematical Morphology, PSL Research University, Rue St Honoré 35, 77300 Fontainebleau, France

L. Holzer

Institute of Computational Physics, ZHAW Winterthur, Wildbachstrasse 21, 8400 Winterthur, Switzerland
} 


\section{Introduction}

For many materials, the microstructure has a strong impact on their effective properties. In particular, this includes functional materials as, e.g., electrodes of solid oxide fuel cells (SOFC) (Suzuki et al., 2009), batteries (Tjaden et al., 2018) and organic photovoltaic devices (Brabec et al., 2011). In order to study the microstructure influence on effective macroscopic properties, numerical simulations based on stochastic microstructure modeling have become a commonly used and powerful tool (Torquato, 2013). Using methods of stochastic geometry (Chiu et al., 2013; Matheron, 1975), microstructures are modeled by random sets to simulate virtual, but realistic microstructures on the computer. These virtual structures can be used as input for numerical simulations of effective macroscopic properties like effective conductivity or permeability. Therefore microstructure-property relationships can be efficiently studied (Scholz et al., 2015; Stenzel et al., 2016), as model-based simulations of virtual microstructures allow for the generation of a large database of various microstructures in short time. Besides studying microstructure-property relationships, fitting parametric stochastic microstructure models to experimental image data has further advantages. In Westhoff et al. (2015), relationships between production parameters and microstructure characteristics have been investigated by the aid of a stochastic model which has been fitted to the microstructure of organic semiconductor films for different values of production parameters. By interpolation of model parameters, virtual microstructures with production parameters which have not been manufactured in reality so far have been generated via predictive simulations. Moreover, having fitted a stochastic model to image data of a microstructure without any structural gradient, the size of the sampling window in which the virtual microstructures are generated is only restricted by computational power. Thus, in most cases virtual microstructures which are larger than the observed ones can be generated. Doing so, a model-based investigation of the local heterogeneity of microstructures can be performed, in particular the size of the representative volume element can be determined as, e.g., in Abdallah et al. (2015); Kanit et al. (2003).

While many different types of stochastic microstructure models are available in the literature for two-phase materials, see e.g., Chiu et al. (2013) and the references therein, less models are present for three-phase microstructures. Diverse packing algorithms have been developed to model both solid phases by a union of spherical (Cai et al., 2011; Kenney et al., 2009), cylindrical or ellipsoidal (Bertei et al., 2014) particles. Furthermore, using excursion sets of two Gaussian random fields allows to reproduce more complex shapes in the model, see e.g. Moussaoui et al. (2018). A detailed theoretical description of this model is given in Chapter 16.2 of Lantuéjoul (2013). In Abdallah et al. (2016), a general method is proposed which shows a way how to model three-phase microstructures based on models for twophase microstructures, more precisely, by two independent random sets. In particular, in Abdallah et al. (2016), those cases are discussed in which both of the two independent random sets are given either by a Boolean model (Molchanov, 1997) or by excursion sets of Gaussian random fields. The latter model type is called the pluri-Gaussian model in the following. It is a special type of the model considered in Moussaoui et al. (2018) and the relationships between its model parameters and microstructure characteristics are well understood. Recently, a further three-phase microstructure model-conceptually different from the previous ones-has been in- 
troduced in Neumann et al. (2016). This model is based on random geometric graphs and has been developed to model three-phase microstructures in SOFC.

In the papers mentioned above, estimation of model parameters and model simulations are described provided that the model type is given. However, in practical applications the choice of the model type is also of significant importance. Thus, in the present paper, we compare the graph-based model proposed in Neumann et al. (2016) with the pluri-Gaussian model of Abdallah et al. (2016). Therefore, we intend to provide advice to practitioners who have to make the decision on model types. The comparison is performed on three-phase microstructures in SOFC anodes. In particular, the models are compared regarding effective transport properties, i.e., effective conductivities in the solid phases and permeability in the pore space. Therefore, effective properties are numerically simulated using FFT-methods (Moulinec and Suquet, 1994; Willot et al., 2014) and the obtained results are interpreted in combination with microstructure characteristics. For the considered microstructures, the goodness-of-fit of prediction formulas for effective conductivity and permeability (Holzer et al., 2017; Stenzel et al., 2016) is discussed. Besides comparing the two models, we derive new results regarding quantitative relationships between model parameters and microstructure characteristics for both models. Based on a simulation study, an empirical formula is found which relates model parameters to volume fractions of phases in the graphbased model. Moreover, an analytical expression for the expected length of the triple phase boundary per unit volume in terms of model parameters is derived for the pluri-Gaussian model. The length of the triple phase boundary is an important characteristic for the considered three-phase microstructures in SOFC anodes as the electrochemical reactions being important for the overall performance are taking place there (Prakash et al., 2014).

The present paper is organized as follows. We start with a description of the considered image data in Section 2, which is used as an example to compare the stochastic microstructure models. The considered microstructure models are described in Section 3, where new results regarding relationships between model parameters and microstructure characteristics are presented. In Section 4, the fit of the models to tomographic image data is discussed with respect to transport relevant microstructure characteristics and numerically simulated effective transport properties. We provide a general comparison of the considered stochastic microstructure models in Section 5, before conclusions are drawn in Section 6 .

\section{Tomographic Image Data of SOFC Anodes}

The $3 \mathrm{D}$ image data to which the stochastic microstructure models are fitted in the present paper represents the microstructure of anodes in solid oxide fuel cells. The anodes consist of nickel, a ceramic phase, called Yttrium-stabilized zirconia (YSZ), and pores. A comprehensive analysis of such anodes based on image data, consisting of cubic voxels with a side length of $30 \mathrm{~nm}$ and obtained by FIB-SEM tomography, is given in Pecho et al. (2015). In this paper, the authors investigated anodes manufactured with different powders of YSZ, i.e., fine, medium and coarse, before and after redox cycling.

In this kind of fuel cells, anodes contribute to electricity generation in the following way, which is illustrated in Figure 1. Oxygen ions are transported through 

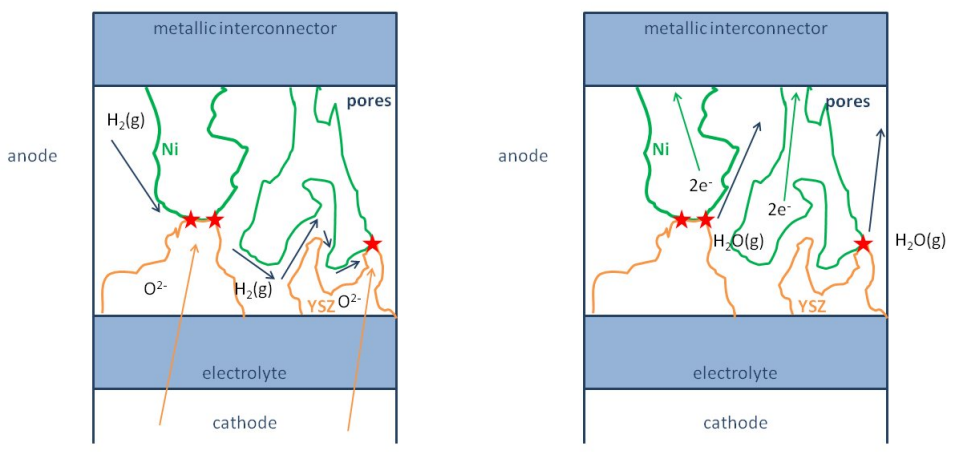

Fig. 1 Schematic sketch of an operating nickel-YSZ anode: oxygen ions and hydrogen are transported to the triple phase boundary (left). Fuel oxidation at the triple phase boundary generates electrons, which are then transported through the nickel phase to the metallic interconnector (right).

the YSZ phase to the triple phase boundary, to which hydrogen is transported through the pores. At the triple phase boundary, the chemical reaction

$$
\mathrm{H}_{2}(\mathrm{~g})+\mathrm{O}^{2-} \rightleftharpoons 2 \mathrm{e}^{-}+\mathrm{H}_{2} \mathrm{O}(\mathrm{g})
$$

takes place resulting in free electrones. These electrones are finally transported to the metallic interconnector. The kinetics of the described reaction as well as the associated polarization and transport resistances depend strongly on the geometry of the nickel-YSZ microstructure, which influences conduction processes in the solid phases and flow in the pore space.

In the present paper, we consider image data from Pecho et al. (2015) corresponding to the fine-structured anode before redox cycling to give an example for fitting two different microstructure models with respect to effective transport properties. Note that in this microstructure all three phases are nearly completely connected. The image represents a cutout of the anode of size $20 \mu \mathrm{m} \times 25 \mu \mathrm{m} \times 15 \mu \mathrm{m}$ and a scaling is performed to remove anisotropy effects, which are ascribed to FIBSEM imaging (Neumann et al., 2016). A 3D visualization of the data set is given in Figure 3 in Section 3.4, where image data is compared to virtual structures simulated by means of stochastic 3D modeling.

\section{Stochastic Microstructure Modeling}

In this section, we give a brief summary of microstructure characteristics which are used for estimation of model parameters and model validation. Then, we recall the definitions of the stochastic microstructure models considered in this paper and present new results regarding the relationship of model parameters and microstructure characteristics. Finally, both models are fitted to $3 \mathrm{D}$ image data. In the following, we consider the three phases as random closed sets (Chiu et al., 2013), where $\Xi_{1}$ denotes the nickel phase, $\Xi_{2}$ the YSZ phase and $\Xi_{3}$ the pore 
space. ${ }^{1}$ Note that both stochastic microstructure models allow to appropriately reproduce specific microstructure characteristics of the considered Ni-YSZ anodes, even if they do not intend to mimic the underlying production process.

\subsection{Microstructure Characteristics}

In both, the graph-based model and the pluri-Gaussian model, the three phases are represented by stationary and isotropic random closed sets $\Xi_{1}, \Xi_{2}$ and $\Xi_{3}$ in $\mathbb{R}^{3}$. In this section we briefly summarize the definitions of microstructure characteristics considered in the present paper. To begin with, the volume fractions $\varepsilon_{1}, \varepsilon_{2}, \varepsilon_{3}$ are defined by

$$
\varepsilon_{i}=\mathbb{E} \nu_{3}\left(\Xi_{i} \cap[0,1]^{3}\right)
$$

for $i=1,2,3$, where $\nu_{3}$ denotes the three-dimensional Lebesgue measure. The specific surface area of phases $i=1,2,3$ is defined by

$$
S_{i}=\mathbb{E} \mathcal{H}_{2}\left(\partial \Xi_{i} \cap[0,1]^{3}\right),
$$

where $\partial A$ denotes the boundary of a set $A \subset \mathbb{R}^{3}$ and $\mathcal{H}_{k}$ denotes the $k$-dimensional Hausdorff measure for each $k=1,2,3$. Moreover, we define the expected length of the triple phase boundary per unit volume by

$$
L_{\mathrm{TPB}}=\mathbb{E} \mathcal{H}_{1}\left(\Xi_{1} \cap \Xi_{2} \cap \Xi_{3} \cap[0,1]^{3}\right) .
$$

Note that the triple phase boundary is of special importance in solid oxide fuel cell anodes as the electrochemical reactions influencing the polarization resistance of the anode half-cell take place there. To quantify the dependency structure of the three phase, we consider the two-point coverage probability functions

$$
C_{i}(h)=\mathbb{P}\left(o \in \Xi_{i}, t \in \Xi_{i}\right),
$$

for $i=1,2,3$, where $|t|=h$. Due to the assumption of isotropy, $C_{i}(h)$ does not depend on the specific choice of $t$, but only on the distance $h$ of $t$ from the origin. The two-point coverage probability functions play an important role for parameter estimation of the pluri-Gaussian model, see Section 3.3. For estimating the parameters of the graph-based model, mean geodesic tortuosities $\tau_{1}, \tau_{2}, \tau_{3}$ measuring the mean length of shortest transportation paths with respect to the materials thickness as well as constrictivities $\beta_{1}, \beta_{2}, \beta_{3}$ quantifying the strength of bottleneck effects are used. Constrictivity for complex microstructures is defined by $\beta=r_{\min }^{2} / r_{\max }^{2}$, where, roughly speaking, $r_{\max }$ is defined as the median of the volume equivalent particle radius distribution and $r_{\min }$ is defined as a median describing the characteristic bottleneck of the microstructure (Holzer et al., 2013). Constrictivity is a value between 0 and 1 . The lower the constrictivity, the stronger is the limiting effect of bottlenecks on transport within the considered phase. For a formal definition of both, mean geodesic tortuosity and constrictivity, in the framework of stationary random closed sets the reader is referred to Neumann et al. (2019).

\footnotetext{
1 A different notation has been used in Neumann et al. (2016), where $\Xi_{1}$ denoted the pore space and $\Xi_{3}$ the nickel phase. We change the notation here to be consistent with the notation of the pluri-Gaussian model considered in Abdallah et al. (2016). In the graph-based model, the index of $\Xi$ is not meaningful in contrast to the pluri-Gaussian model.
} 


\subsection{Graph-based Microstructure Model}

In the graph-based model (GBM), introduced in Neumann et al. (2016), a stationary random geometric graph is considered for each phase. The graphs are random in the sense that their vertex sets are modeled by random point processes. The edges of the graphs are put deterministically according to a parametric rule. Finally, three full-dimensional phases are constructed from the random graphs. Formally, the three closed random sets $\Xi_{1}, \Xi_{2}$ and $\Xi_{3}$ are defined as follows. Let $X_{1}, X_{2}$ and $X_{3}$ be independent homogeneous Poisson point processes (Møller and Waagepetersen, 2004) with some intensities $\lambda_{1}, \lambda_{2}, \lambda_{3}>0$, which model the vertex sets of the random geometric graphs. That is, the vertices are distributed completely at random in the three-dimensional space with a predefined expected number of points in the unit cube. Then, the corresponding graphs $\mathcal{G}_{1}, \mathcal{G}_{2}, \mathcal{G}_{3}$ are obtained by putting the edges according to the rule of the so-called betaskeletons (Kirkpatrick and Radke, 1985) with parameters $b_{1}, b_{2}, b_{3} \geq 1$, respectively. For $i=1,2,3$, connectivity properties of $\mathcal{G}_{i}$ can be controlled by the aid of the parameter $b_{i}$. The higher the value of $b_{i}$ is, the less edges are put in the graph $\mathcal{G}_{i}$. In particular, for $1 \leq b_{i} \leq 2$, the graph $\mathcal{G}_{i}$ is completely connected with probability 1 as stated in Hirsch et al. (2013). Given the graphs $\mathcal{G}_{i}$ and three additional model parameters $\gamma_{1}, \gamma_{2}, \gamma_{3}>1$, we define the random closed sets

$$
\Xi_{i}=\left\{x \in \mathbb{R}^{3}: d_{\gamma_{i}}^{\prime}\left(x, \mathcal{G}_{i}\right) \leq \min _{1 \leq j \leq 3} d_{\gamma_{j}}^{\prime}\left(x, \mathcal{G}_{j}\right)\right\}, \quad i=1,2,3
$$

where $d_{\gamma_{i}}^{\prime}\left(x, \mathcal{G}_{i}\right)=\min \left\{\gamma_{i} d\left(x, \mathcal{G}_{i}\right), d\left(x, X_{i}\right)\right\}$ and $d(x, A)=\inf _{y \in A}|x-y|$ is the minimum Euclidean distance between $x$ and a set $A \subset \mathbb{R}^{3}$. This means that a point $x$ belongs to, e.g., $\Xi_{1}$ if the distance of $x$ to $\mathcal{G}_{1}$ with respect to $d_{\gamma_{1}}^{\prime}$ does not exceed the distance of $x$ to $\mathcal{G}_{2}$ and $\mathcal{G}_{3}$ with respect to $d_{\gamma_{2}}^{\prime}$ and $d_{\gamma_{3}}^{\prime}$, respectively. Note that $d_{1}^{\prime}(x, A)=d(x, A)$ for all $x \in \mathbb{R}^{3}, A \subset \mathbb{R}^{3}$. Finally a Gaussian kernel with some variance $s_{\text {GBM }}^{2}$ is applied to smooth the boundaries between the three phases as described in Neumann et al. (2016). Note that the resulting random sets $\Xi_{1}, \Xi_{2}$ and $\Xi_{3}$ are stationary as well as isotropic. For the simulation of model realizations of the GBM, we refer to Section 3.2.1 in Neumann et al. (2016). Details regarding the implementation and the runtime of simulations in the present paper are provided in "Appendix C".

In the following, we extend Equation (3.10) in Neumann et al. (2016), which relates model parameters to the volume fractions $\varepsilon_{1}, \varepsilon_{2}, \varepsilon_{3}$ of the random closed sets $\Xi_{1}, \Xi_{2}, \Xi_{3}$ in the case $\gamma_{1}=\gamma_{2}=\gamma_{3}=1$. To be more precise, we derive an approximation formula for $\varepsilon_{1}, \varepsilon_{2}, \varepsilon_{3}$ in the more general case $\gamma_{1}=\gamma_{2}=\gamma_{3}=\gamma$ and $s_{\mathrm{GBM}}=0.02 \mu \mathrm{m}$ by a simulation study, where $\gamma>1$. We choose $s_{\mathrm{GBM}}=$ $0.02 \mu \mathrm{m}$ since this value was obtained when fitting the model to tomographic image data, described in Section 2, cf. also Table 1. For this purpose, volume fractions are estimated for all parameter vectors $\left(\lambda_{1}, \lambda_{2}, \lambda_{3}, b_{1}, b_{2}, b_{3}, \gamma\right) \in \Theta$, where $\Theta=$

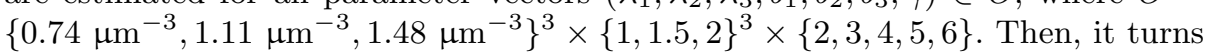
out that for each $i \in\{1,2,3\}$

$$
\widehat{\varepsilon}_{i}^{\star}=\frac{h\left(\lambda_{i}, b_{i}, \gamma\right)}{\sum_{j=1}^{3} h\left(\lambda_{j}, b_{j}, \gamma\right)},
$$




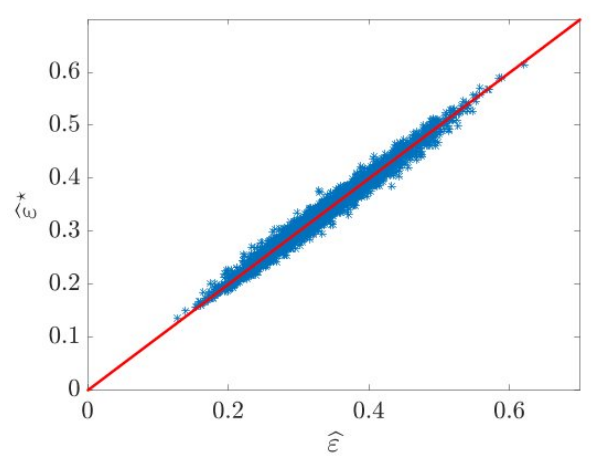

Fig. 2 Plot of volume fractions $\widehat{\varepsilon}^{\star}$ estimated by Equation (6) over volume fractions estimated $\widehat{\varepsilon}$ by the point-count method. For each parameter constellation, the values $\widehat{\varepsilon}^{\star}$ and $\widehat{\varepsilon}$ are computed for all three phases.

where

$$
h(\lambda, b, \gamma)=2 \lambda \gamma^{2}+\sqrt[3]{\frac{\lambda^{2}}{(3 b-1)^{4}}}
$$

for all $\lambda>0, b \geq 1, \gamma>1$, leads to an appropriate approximation formula of volume fractions.

Figure 2 shows that the approximation by $\widehat{\varepsilon}^{\star}$ given by Equation (6) leads to similar results as the estimator $\widehat{\varepsilon}$ obtained by using the point-count method(Chiu et al., 2013) to estimate volume fractions from discretized model realizations. The coefficient of determination $R^{2}$ is given by $R^{2}=0.9806$.

\subsection{Pluri-Gaussian Microstructure Model}

A different model for the stationary random closed sets $\Xi_{1}, \Xi_{2}, \Xi_{3}$, namely the pluri-Gaussian model (PGM) (Abdallah et al., 2016), is based on excursion sets of Gaussian random fields in $\mathbb{R}^{3}$, see Chiu et al. (2013); Lantuéjoul (2013). A Gaussian random field $Z=\left\{Z(t), t \in \mathbb{R}^{3}\right\}$ in $\mathbb{R}^{3}$ is a random function mapping from $\mathbb{R}^{3}$ to $\mathbb{R}$, where for each finite collection of points $t_{1}, \ldots, t_{n} \in \mathbb{R}^{3}, n \geq 1$, the random vector $\left(Z\left(t_{1}\right), \ldots, Z\left(t_{n}\right)\right)$ follows the law of a multivariate normal distribution. For an introduction to random fields and their geometric properties, we refer to Adler (1981). Let $Z=\left\{Z(t), t \in \mathbb{R}^{3}\right\}$ and $Y=\left\{Y(t), t \in \mathbb{R}^{3}\right\}$ be two independent motion-invariant, i.e., stationary and isotropic, Gaussian random fields with $Z(o), Y(o) \sim \mathcal{N}(0,1)$ and covariance functions $\rho_{Z}$ and $\rho_{Y}$, respectively. Here $o$ denotes the origin in $\mathbb{R}^{3}$. Note that for each $h \geq 0$, the value of the covariance function $\rho_{Z}(h)$ is defined as the covariance of the random variables $Z(o)$ and $Z(t)$, where $t$ has distance $h$ to the origin. Due to the stationarity and isotropy of $Z$, the value $\rho_{Z}(h)$ does not depend on the particular choice of $t$. Let $u_{Z}, u_{Y} \in \mathbb{R}$ be arbitrary real numbers and define the random closed sets $\Xi_{1}, \Xi_{2}$ and $\Xi_{3}$ by $\Xi_{1}=\left\{t \in \mathbb{R}^{3}: Z(t) \geq u_{Z}\right\}, \quad \Xi_{2}=\left\{t \in \mathbb{R}^{3}: Z(t) \leq u_{Z}, Y(t) \geq\right.$ $\left.u_{Y}\right\}$ and $\Xi_{3}=\left\{t \in \mathbb{R}^{3}: Z(t) \leq u_{Z}, Y(t) \leq u_{Y}\right\}$. This means that the three phases are defined according to the values of the random fields $Z$ and $Y$, e.g., $\Xi_{1}$ contains all points in $\mathbb{R}^{3}$, where the value of the random field $Z$ exceeds the 
threshold $u_{Z}$. Due to the stationarity and isotropy of the random fields $Z$ and $Y$, the random sets $\Xi_{1}, \Xi_{2}$ and $\Xi_{3}$ are stationary and isotropic as well. For the PGM, some relationships between model parameters and structural characteristics as volume fractions, two-point coverage probability functions and specific surface areas are well understood (Abdallah et al., 2016). By definition, we have $\varepsilon_{1}=$ $\Phi^{-1}\left(1-u_{Z}\right), \varepsilon_{2}=\left(1-\varepsilon_{1}\right) \Phi^{-1}\left(1-u_{Y}\right)$ and $\varepsilon_{3}=1-\varepsilon_{1}-\varepsilon_{2}$, which allows for an estimation of $u_{Z}$ and $u_{Y}$ by estimating volume fractions from image data of the considered microstructures. Moreover, for all $h \geq 0$, the following equations

$$
C_{1}(h)=\varepsilon_{1}^{2}+\frac{1}{2 \pi} \int_{0}^{\rho_{Z}(h)} \frac{e^{\frac{-u_{Z}^{2}}{1+t}}}{\sqrt{1-t^{2}}} \mathrm{~d} t
$$

see Lantuéjoul (2013), and

$$
\frac{C_{2}(h)}{1-2 p_{1}+C_{1}(h)}=\left(\frac{\varepsilon_{2}}{1-\varepsilon_{1}}\right)^{2}+\frac{1}{2 \pi} \int_{0}^{\rho_{Y}(h)} \frac{e^{\frac{-u_{Y}^{2}}{1+t}}}{\sqrt{1-t^{2}}} \mathrm{~d} t,
$$

see Abdallah et al. (2016), hold. They relate the covariance functions $\rho_{X}$ and $\rho_{Y}$ to the two-point coverage probability functions of the three phases. We fit a parametric model to the two-point coverage probability functions $C_{1}$ and $C_{2}$, which can be estimated from image data. Therefore, we choose a different class of parametric functions than the one considered in Abdallah et al. (2016). To be more precise, we use the model

$$
C_{i}(h)=\varepsilon_{i}^{2}+\varepsilon_{i}\left(1-\varepsilon_{i}\right) e^{-\theta_{i 1} h\left(1+\theta_{i 2} h\right)},
$$

for $h \geq 0, i \in\{1,2\}$ and some parameters $\theta_{11}, \theta_{12}, \theta_{21}, \theta_{22}>0$. Then, we use Equations (8) and (9) for a numerical computation of $\rho_{Z}$ and $\rho_{Y}$. Since, for each $h \geq 0$, the right-hand sides in Equations (8) and (9) are monotonously increasing in $\rho_{Z}(h)$ and $\rho_{Y}(h)$, respectively, the values of $\rho_{Z}(h)$ and $\rho_{Y}(h)$ can be computed using the method of bisection. In a further step, $\rho_{Z}$ and $\rho_{Y}$ are smoothed by the aid of a Gaussian kernel with some variance $s_{\mathrm{PGM}}^{2}$ to minimize the errors with respect to specific surface areas of the three phases (Abdallah et al., 2016). We refer to Section 7 in Abdallah et al. (2016) for a description of how to simulate realizations of the PGM. Details regarding the implementation and runtime of simulations performed in the present paper are given in "Appendix C".

In the PGM, the influence of model parameters on contact areas between any two of the three phases is well understood (Abdallah et al., 2016). Besides contact areas, the length of the triple phase boundary is important for the functionality of certain microstructures, as, e.g., for the Ni-YSZ anodes considered in the present paper. We give a result which relates the expected length of the triple phase boundary $L_{\mathrm{TPB}}$ to the covariance functions $\rho_{Z}$ and $\rho_{Y}$ in the case that $Z$ and $Y$ are mean square differentiable (Adler, 1981). In the following, we denote by $f(0+)$ the derivative from the right at 0 of a function $f:[0, \infty) \longrightarrow \mathbb{R}$, provided that it exists.

Proposition 1 Let $Z$ and $Y$ be mean square differentiable. Then, the expected length of triple phase boundary per unit volume is given by

$$
L_{\mathrm{TPB}}=\frac{e^{-\left(u_{Z}^{2}+u_{Y}^{2}\right) / 2}}{\pi} \sqrt{\rho_{Z}^{\prime \prime}(0+) \rho_{Y}^{\prime \prime}(0+)} .
$$


The corresponding proof is postponed to "Appendix A". Note that the derivatives in Equation (11) exist and are finite due to the assumption that $Z$ and $Y$ are mean square differentiable (Adler, 1981). Moreover, using the spectral representation of isotropic covariance functions (Adler, 1981, Theorem 2.5.3) it can be easily shown that $\rho_{Z}^{\prime \prime}(0+), \rho_{Y}^{\prime \prime}(0+)<0$ and thus, $\sqrt{\rho_{Z}^{\prime \prime}(0+) \rho_{Y}^{\prime \prime}(0+)}$ is well defined. Note that in our case the functions $\rho_{Z}$ and $\rho_{Y}$ are only implicitly determined by Equations (8)(10) and the smoothing by a Gaussian kernel. As we focus on effective transport properties of the three phases for a comparison of the graph-based model with the pluri-Gaussian model, a detailed analysis of the length of the triple phase boundary is beyond the scope of the present paper and is left to future research. This includes an investigation of the relationship between the analytically derived expected length of the triple phase boundary per unit volume with values computed from discrete image data.

\subsection{Model Fitting to 3D Image Data of SOFC Anodes}

In this section we describe the fitting of model parameters to the tomographic image data presented in Section 2. Model validation is postponed to Section 4.3, when effective properties like effective conductivities of solids and permeability of the pore space are computed for tomographic image data and model realizations. The model parameters of the graph-based model have already been fitted to this data set in Neumann et al. (2016). The model parameters are chosen in order to minimize the discrepancy between tomographic image data and model realizations regarding volume fractions, mean geodesic tortuosities and constrictivities of the solid phases as well as the occurrence of triple phase boundary voxels. The minimization is iteratively performed using the Nelder-Mead algorithm (Nelder and Mead, 1965). The numerical values of the fitted model parameters are given in Table 1 and a model realization with fitted parameters is visualized in Figure 3.

Table 1 Numerical values of fitted model parameters of the graph-based model.

\begin{tabular}{cccccccccc}
$\lambda_{1}$ & $\lambda_{2}$ & $\lambda_{3}$ & $b_{1}$ & $b_{2}$ & $b_{3}$ & $\gamma_{1}$ & $\gamma_{2}$ & $\gamma_{3}$ & $s_{\mathrm{GBM}}$ \\
\hline $0.95 \mu \mathrm{m}^{-3}$ & $1.18 \mu \mathrm{m}^{-3}$ & $0.87 \mu \mathrm{m}^{-3}$ & 1.94 & 1.97 & 2.11 & 4.12 & 4.31 & 4.47 & $0.02 \mu \mathrm{m}$
\end{tabular}

Table 2 Numerical values of fitted model parameters of the pluri-Gaussian model.

\begin{tabular}{ccccccc}
$\varepsilon_{1}$ & $\varepsilon_{2}$ & $\theta_{11}$ & $\theta_{12}$ & $\theta_{21}$ & $\theta_{22}$ & $s_{\text {PGM }}$ \\
\hline 0.33 & 0.42 & $2.00 \mu \mathrm{m}^{-1}$ & $1.10 \mu \mathrm{m}^{-1}$ & $2.33 \mu \mathrm{m}^{-1}$ & $0.67 \mu \mathrm{m}^{-1}$ & $0.11 \mu \mathrm{m}$
\end{tabular}

The parameters of the pluri-Gaussian model are fitted proceeding analogously to Abdallah et al. (2016). At first, the discrete FFT is used to estimate the twopoint coverage probability functions $C_{1}$ and $C_{2}$ from image data, cf. Ohser and Schladitz (2009). The functions $C_{1}$ and $C_{2}$ are fitted by the parametric functions given in Equation (10). The estimated numerical values of $\theta_{11}, \theta_{12}, \theta_{21}$ and $\theta_{22}$ are given in Table 2. In the next step, the fitted parametric functions are plugged 




(a)

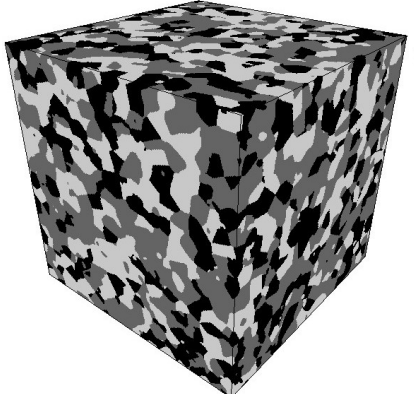

(b)

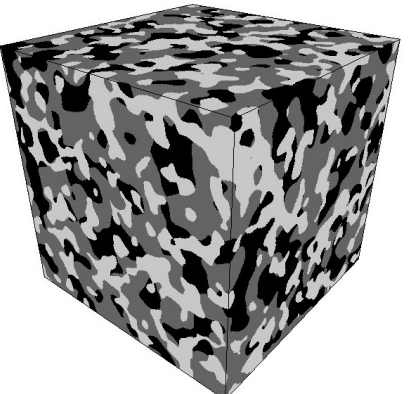

(c)

Fig. 3 3D cutout of tomographic image data (a) and virtual microstructures simulated with the graph-based model (b) and with the pluri-Gaussian model (c). The microstructures consist of pores (black), YSZ (dark grey) and nickel (bright grey). Each cutout has a size of $12 \mu \mathrm{m} \times$ $12 \mu \mathrm{m} \times 12 \mu \mathrm{m}$ and consists of cubic voxels with an edge length of $30 \mathrm{~nm}$.

into Equations (8) and (9) to numerically compute $\rho_{Z}$ and $\rho_{Y}$. For this purpose, the method of bisection is used. This is possible since the right-hand sides in Equations (8) and (9) are monotonous in $\rho_{Z}(h)$ (and $\rho_{Y}(h)$ respectively) for each fixed $h \geq 0$.

Simulations of the fitted pluri-Gaussian model show that the expected area of interfaces per unit volume is overestimated compared to the values estimated from image data. For the estimation of the expected area of interfaces, the method presented in Ohser and Schladitz (2009) is used. Thus, the smoothing parameter $s_{\text {PGM }}$ is fitted in order to minimize the difference between interface areas of model realizations and image data. By means of a simulation study, we obtain $s_{\mathrm{PGM}}=$ $0.11 \mu \mathrm{m}$. A realization of the fitted pluri-Gaussian model is visualized in Figure 3.

\section{Effective Transport Properties}

To validate the stochastic microstructure models considered in Section 3, effective transport properties are numerically simulated for model realizations and tomographic image data, followed by a discussion of microstructure-property relationships. At first, the numerical simulation of conductivity and permeability is briefly described. For further information with respect to implementation and runtime of the numerical simulations, the reader is referred to Appendix C.

\subsection{Numerical Simulation of Effective Transport Properties}

The fluid flow occurring in the pore phase is modeled using the Stokes equation for an incompressible Newtonian fluid with viscosity $\mu_{f}$ and velocity $\mathbf{v}$ driven by a pressure field $p$ :

$$
\mu_{f} \Delta \mathbf{v}=\nabla p, \quad \nabla \cdot \mathbf{v}=0,
$$

where $\Delta=\nabla \cdot \nabla$ is the Laplacian operator, here applied to each component of $\mathbf{v}, \nabla p$ the gradient vector of the pressure field, and $\nabla \cdot \mathbf{v}$ the divergence of the 
velocity field. The problem's boundary conditions read:

$$
\mathbf{v}(\mathbf{x}) \equiv 0, \quad \mathbf{x} \in \Gamma, \quad\langle\nabla p\rangle=\mathbf{G}, \quad \mathbf{v}(\mathbf{x}) \#, \quad p(\mathbf{x})-\mathbf{G} \cdot \mathbf{x} \#,
$$

where $\Gamma$ is the pore-solid interface, $\langle\cdot\rangle$ denotes mean over the computational domain, $\mathbf{G}$ is a vector representing the macroscopically applied pressure drop and \# denotes periodicity over the computational domain. The effective response of the material is given by Darcy's equation:

$$
\mu_{f}\left\langle v_{i}\right\rangle=\kappa_{i j} G_{j}, \quad \mathbf{G}=\langle\nabla p\rangle,
$$

where $\kappa$ is the permeability of the medium and $\mathbf{G}$ the macroscopic pressure gradient. For isotropic or quasi-isotropic media, as considered in the present paper, the second-order tensor $\kappa$ reduces to a scalar. Full-field solutions for the velocity and pressure fields are computed numerically on digitalized images using the Fourier-based "FFF-Stokes" algorithm (Wiegmann, 2007). For convenience, we set $G_{1}=1, G_{2}=G_{3}=0, \mu_{f}=1$ (arbitrary units) and compute the permeability $\kappa_{\text {pores }}=\kappa_{11}=\left\langle\mu_{f} v_{1} / G_{1}\right\rangle$. Note that $\kappa_{\text {pores }}$ is an intrinsic quantity that does not depend on $\mu_{f}$ but solely on the geometry.

Making use of the Fourier-based "direct scheme" with "discrete" Green operator (Willot et al., 2014), we estimate ionic and electronic conductivity in the two solid phases. We solve the following problem:

$$
\nabla \cdot \mathbf{J}=0, \quad \mathbf{E}=-\nabla \phi \quad \mathbf{J}(\mathbf{x})=\left\{\begin{array}{cc}
\sigma_{\mathrm{sol}} \mathbf{E}(\mathbf{x}), & \text { if } \mathbf{x} \in \mathcal{S} \\
0, & \text { otherwise }
\end{array}\right.
$$

where $\mathcal{S}$ denotes the considered conductive phase, $\mathbf{J}$ is the electrical current vector (or particle current), E the electrical vector field (or opposite gradient of ionic concentration), $\phi$ is the electrical potential (or ionic concentration) and $\sigma_{\text {sol }}$ is the intrinsic electrical conductivity of nickel (or the intrinsic ionic diffusion coefficient of YSZ). The periodic boundary conditions read:

$$
\mathbf{J}(\mathbf{x}) \#, \quad \mathbf{E}(\mathbf{x}) \#, \quad \phi(\mathbf{x})+\langle\mathbf{E}\rangle \cdot \mathbf{x} \#,
$$

where $\langle\mathbf{E}\rangle$ denotes the applied electrical field (or applied concentration gradient). In both problems, the effective conductivity $\sigma$ is computed by averaging the fields $\mathbf{E}$ and $\mathbf{J}$ :

$$
\langle\mathbf{J}\rangle=\sigma \cdot\langle\mathbf{E}\rangle .
$$

Again, the second-order tensor $\sigma$ reduces to a scalar for isotropic media. We apply $\left\langle E_{1}\right\rangle=1,\left\langle E_{2}\right\rangle=\left\langle E_{3}\right\rangle=0$ and define the normalized effective conductivity (or $M$-factor) related to the nickel and YSZ phases by $\sigma_{\mathrm{Ni}, \mathrm{YSZ}}=\sigma_{11} / \sigma_{\mathrm{sol}}$, where $\sigma_{11}=\left\langle J_{1}\right\rangle /\left\langle E_{1}\right\rangle$, computed in their respective problems. The $M$-factor does not depend on $\sigma_{\text {sol }}$, but only on the geometry of the two solid phases.

Boundary conditions are applied such that the solutions for the conductivity and Stokes flow problems are that of a periodic, infinite 3D medium with elementary cell given by the PGM or GBM models, simulated on finite-size domains containing $512^{3}$ voxels, or to tomographic 3D image data, containing $849 \times 648 \times 430$ voxels. Opposite faces of the latter do not correspond as in the elementary cell of a periodic structure, which induces unwanted boundary effects along the surfaces. To minimize such effects, we insert a layer with a size of 50 voxels of pore (or conducting phase) normal to the direction of the applied loading, which serves 


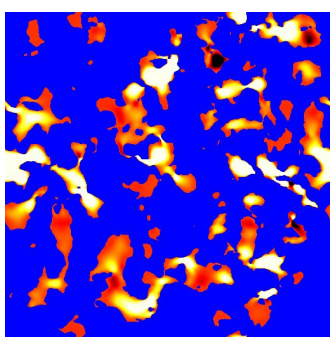

(a)

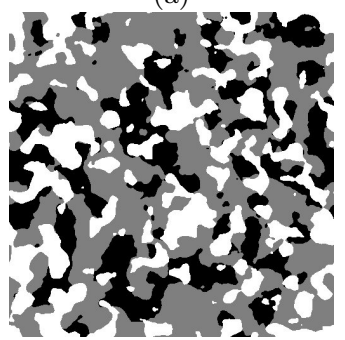

(d)

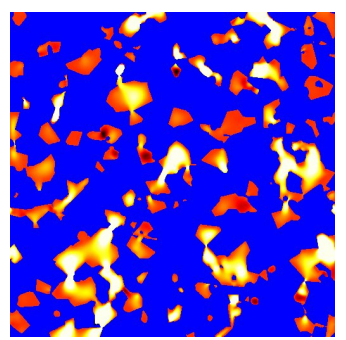

(b)

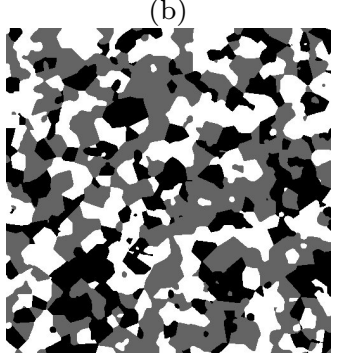

(e)

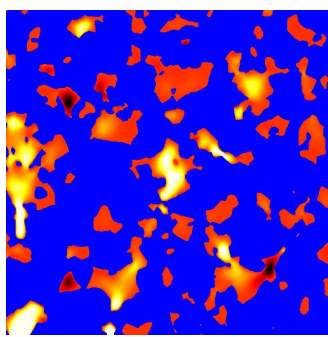

(c)

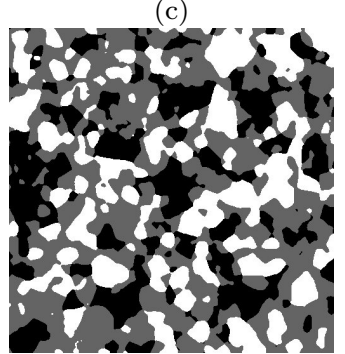

(f)

Fig. 4 FFT maps of the flow velocity component $v_{1}$, oriented parallel to the applied pressure drop $G_{1}=1(\mathrm{~Pa})$ and corresponding microstructure with pores in black (2D sections, axis 1 , i.e. the transport direction, is oriented vertically, top to bottom). The $2 \mathrm{D}$ sections correspond to realizations of the optimized PGM model (a,d) and the optimized GBM model (b,e) as well as to tomographic image data $(\mathrm{c}, \mathrm{f})$. The same color scale in the range $[-1 ; 1.2]\left(\times 10^{-5} \mathrm{~ms}^{-1}\right)$ is used in maps (a-c). Positive values of the fluid velocity in white and yellow, negative values in black, zero values in red (solid phase in blue). The images have a size of $12 \mu \mathrm{m} \times 12 \mu \mathrm{m}$.

to connect the current or fluid flow paths from one side to the opposite. The effective conductivity and permeability are estimated by taking field averages over the interior domain, without the layer. For the FIB-SEM image, this treatment increases the conductivity by about $4 \%$ in the nickel phase and $3 \%$ in the YSZ phase, whereas the permeability in the pore phase is increased by a relative factor of $7 \%$. Figure 4 shows $2 \mathrm{D}$ sections of FFT field maps for the fluid velocity component in the pore phase, for the GBM and PGM models, as well as for the FIB-SEM data, to be compared with the geometry of the underlying microstructures. In all three microstructures, only some of the paths through the pore space intersecting the $2 \mathrm{D}$ sections represented in the figure participate in the fluid transport. Also, some of the paths transport fluid in a direction opposite to the applied pressure drop (black spots), highlighting the tortuosity of the fluid paths.

4.2 Optimization of Model Parameters for Precise Fitting of Effective Transport Properties

As described in Section 3.4 the graph-based model as well as the pluri-Gaussian model are fitted to tomographic image data. By means of numerical simulations, the corresponding effective transport properties $\kappa_{\text {pores }}, \sigma_{\mathrm{Ni}}$ and $\sigma_{\mathrm{YSZ}}$ are computed for tomographic image data and for realizations of both models with the fitted parameter constellations given in Tables 1 and 2. In the following, we denote the virtual structure with fitted parameters by GBM (fit) and PGM (fit) for the 
graph-based model and the pluri-Gaussian model, respectively. Additionally, the parameters of both models are slightly varied and model realizations are simulated. Then, effective transport properties are also computed for these modified virtual microstructures. It turns out that with slightly varied model parameters the fit of effective transport properties can be further improved.

For this purpose, we perform a simulation study. For the graph-based model $s_{\mathrm{GBM}}=0.02 \mu \mathrm{m}$ is kept fix and we restrict ourselves to the simplified case in which $b_{1}=b_{2}=b_{3}=b \in\{1.8,1.9,2.0,2.1\}$ and $\gamma_{1}=\gamma_{2}=\gamma_{3}=\gamma \in\{2$, $3,3.5,4.1,4.2,4.3,4.4,5,6\}$. This simplification is reasonable since the values of $b_{1}, b_{2}, b_{3}$ and $\gamma_{1}, \gamma_{2}, \gamma_{3}$ fitted to tomographic image data are close to each other, cf. Table 1. Moreover, we fix $\lambda_{2}=1.18 \mu^{-3}$ and choose $\lambda_{1}$ and $\lambda_{3}$ in order to fit the volume fractions of tomographic image data. Therefore, we use the relationship between model parameters and volume fractions given in Equation (6). For the pluri-Gaussian model, we keep the parameters $\varepsilon_{1}, \varepsilon_{2}, \theta_{11}, \theta_{21}$ and $s_{\mathrm{PGM}}$ fix and vary the two remaining parameters, i.e., $\theta_{12} \in\{0.17,0.33,1.00,1.67\}\left(\mu \mathrm{m}^{-1}\right)$ and $\theta_{22} \in\{0.33,0.67,2.00,3.33\}\left(\mu \mathrm{m}^{-1}\right)$. Then, for each of the simulated virtual microstructures permeability of the pore space and conductivity of the two solid phases are computed. We denote the virtual structures for which the sum of relative errors regarding effective properties of tomographic image data is minimized by GBM (opt) and PGM (opt), respectively. The optimum parameter constellation is $\beta=1.8, \gamma=2$ in the graph-based model and $\theta_{12}=1.67 \mu \mathrm{m}^{-1}, \theta_{22}=0.33 \mu \mathrm{m}^{-1}$ in the pluri-Gaussian model. The achieved improvement regarding the fit of effective properties is discussed in the following section.

Table 3 Numerically simulated values of effective transport properties $\kappa_{\text {pores }}, M_{\mathrm{Ni}}$ and $M_{\mathrm{YSZ}}$ for tomographic image data and realizations of both models with fitted parameter constellations.

\begin{tabular}{lccc} 
& $\kappa_{\text {pores }}\left(10^{-11} \mathrm{~cm}^{2}\right)$ & $M_{\mathrm{Ni}}$ & $M_{\mathrm{YSZ}}$ \\
\hline Image data & $\mathbf{0 . 4 0 3}$ & $\mathbf{0 . 0 7 5}$ & $\mathbf{0 . 1 7 7}$ \\
\hline GBM (fit) & 0.278 & 0.091 & 0.152 \\
\hline GBM (opt) & 0.353 & 0.097 & 0.175 \\
\hline PGM (fit) & 0.147 & 0.098 & 0.191 \\
\hline PGM (opt) & 0.419 & 0.092 & 0.193
\end{tabular}

Table 4 Estimated values of volume fractions $\varepsilon=\left(\varepsilon_{1}, \varepsilon_{2}, \varepsilon_{3}\right)$, mean geodesic tortuosities $\tau=\left(\tau_{1}, \tau_{2}, \tau_{3}\right)$ and constrictivities $\beta=\left(\beta_{1}, \beta_{2}, \beta_{3}\right)$ for tomographic image data and realizations of both models with fitted parameter constellations. Recall from Section 3 that the index 1 belongs to nickel, 2 to YSZ and 3 to the pore space.

\begin{tabular}{lccc} 
& $\varepsilon$ & $\tau$ & $\beta$ \\
\hline Image data & $\mathbf{( 0 . 3 3 , 0 . 4 2 , 0 . 2 5 )}$ & $\mathbf{( 1 . 1 7 , \mathbf { 1 . 1 0 } , \mathbf { 1 . 2 6 } )}$ & $\mathbf{( 0 . 3 3 , 0 . 4 2 , \mathbf { 0 . 3 1 } )}$ \\
\hline GBM (fit) & $(0.32,0.40,0.28)$ & $(1.13,1.10,1.17)$ & $(0.33,0.44,0.24)$ \\
\hline GBM (opt) & $(0.32,0.42,0.26)$ & $(1.12,1.09,1.15)$ & $(0.34,0.46,0.28)$ \\
\hline PGM (fit) & $(0.34,0.41,0.25)$ & $(1.16,1.08,1.17)$ & $(0.46,0.61,0.39)$ \\
\hline PGM (opt) & $(0.32,0.42,0.26)$ & $(1.14,1.09,1.19)$ & $(0.46,0.57,0.39)$
\end{tabular}


Table 5 Estimated values of $r_{\max }=\left(r_{\max , 1}, r_{\max , 2}, r_{\max , 3}\right), r_{\min }=\left(r_{\min , 1}, r_{\min , 2}, r_{\min , 3}\right)$ for tomographic image data and realizations of both models with fitted parameter constellations. Recall from Section 3 that the index 1 belongs to nickel, 2 to YSZ and 3 to the pore space.

\begin{tabular}{lcc} 
& $r_{\max }\left[10^{-6} \mathrm{~m}\right]$ & $r_{\min }\left[10^{-6} \mathrm{~m}\right]$ \\
\hline Image data & $\mathbf{( 0 . 3 7 4 4}, \mathbf{0 . 3 0 0 0}, \mathbf{0 . 3 0 9 3})$ & $\mathbf{( 0 . 2 1 4 5 , ~ 0 . 1 9 2 0 , 0 . 1 7 3 4 )}$ \\
\hline GBM (fit) & $(0.3285,0.3483,0.3126)$ & $(0.1872,0.2286,0.1533)$ \\
\hline GBM (opt) & $(0.3285,0.3606,0.3048)$ & $(0.1614,0.2454,0.1902)$ \\
\hline PGM (fit) & $(0.3906,0.2109,0.162)$ & $(0.2646,0.1641,0.1011)$ \\
\hline PGM (opt) & $(0.3072,0.3366,0.2898)$ & $(0.20910 .25350 .1806)$
\end{tabular}

\subsection{Model Validation and Microstructure-Property Relationships}

We validate both stochastic microstructure models considered in Section 3 with respect to effective transport properties. Therefore, we consider permeability of the pore space and the $M$-factors of the two solid phases, denoted by $M_{\mathrm{Ni}}$ and $M_{\text {YsZ }}$. The $M$-factor of a conducting phase is defined as the ratio of effective conductivity over intrinsic conductivity. Since in our study the intrinsic conductivity is a material dependent constant, the effective conductivity is directly related to the corresponding $M$-factor. Moreover, the microstructure characteristics volume fraction $\varepsilon$, mean geodesic tortuosity $\tau$ as well as $r_{\min }$ and $r_{\max }$ determining constrictivity $\beta$ are computed for all three phases. These microstructure characteristics have a strong influence on effective conductivity as shown in Stenzel et al. (2016), where the empirical formula

$$
\widehat{M}=\frac{\varepsilon^{1.15} \beta^{0.37}}{\tau^{4.39}}
$$

of the $M$-factor was derived based on a combination of stochastic modeling and numerical simulations. Note that prediction errors of Equation (18) increase for smaller volume fractions with a tendency to overestimate the $M$-factor (Stenzel et al., 2017, Figure 7). In a recent study (Holzer et al., 2017) for gas diffusion layers used in polymer electrolyte fuel cells, the following empirical formula for permeability $\kappa$ has been obtained:

$$
\widehat{\kappa}=\frac{\left(r_{\min }+r_{\max }\right)^{2}}{32} \widehat{M}
$$

In Tables 3, 4 and 5, the computed microstructure characteristics and the simulated effective transport properties are given.

Connectedness, of central importance for transport properties, should be very similar in the models and tomographic images. We emphasize that each phase in both the PGM and GBM models are well-connected as in the FIB-SEM 3D image. More precisely, in the graph-based model, complete connectivity of each phase $i \in\{1,2,3\}$ is theoretically guaranteed if $b_{i} \leq 2$. Even if $b_{3}>2$ here, the largest completely-connected cluster in the pore space takes more than $98.5 \%$ of the pore space in the GBM (fit) (Neumann et al., 2016), whereas this quantity is larger than $99 \%$ for all three phases in both structures realized by the pluriGaussian model, i.e., PGM (fit) and PGM (opt). 
Volume fractions are fitted well by the stochastic microstructure models. Only small discrepancies occur for GBM (fit), see Table 4. The reason for these discrepancies is that the parameter fitting was performed here in order to numerically optimize the fit of the two solid phases with respect to $\varepsilon, \tau, \beta$ and the occurrence of triple phase boundary voxels simultaneously. Thus the fit of volume fractions is not as good as the fit in the cases of GBM (opt), PGM (fit) and PGM (opt), where $\varepsilon_{1}, \varepsilon_{2}$ and $\varepsilon_{3}$ are adjusted due to known relationships between volume fractions and model parameters. Mean geodesic tortuosities behave similarly for both models. While $\tau_{2}$ is fitted well, $\tau_{1}$ and $\tau_{3}$ are slightly underestimated. For constrictivities of nickel and YSZ, the best fit is obtained by GBM (fit), which is not surprising as these values have been used for model fitting. By a variation of model parameters in the graph-based model the fit of $\beta_{1}$ and $\beta_{2}$ becomes slightly worse. However, the accordance of $\beta_{3}$ with respect to tomographic image data is improved. Furthermore, one can observe that constrictivity is overestimated in the pluri-Gaussian model, i.e., there are less limiting effects of bottlenecks in the model realizations compared to tomographic image data.

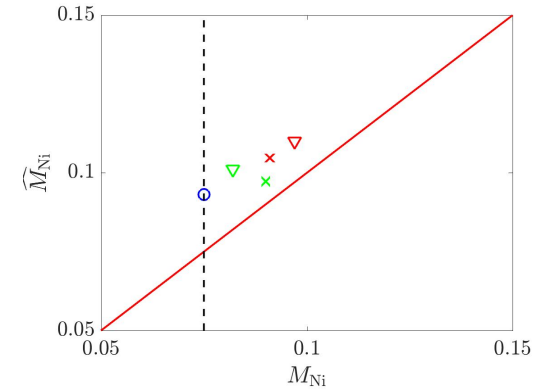

(a)

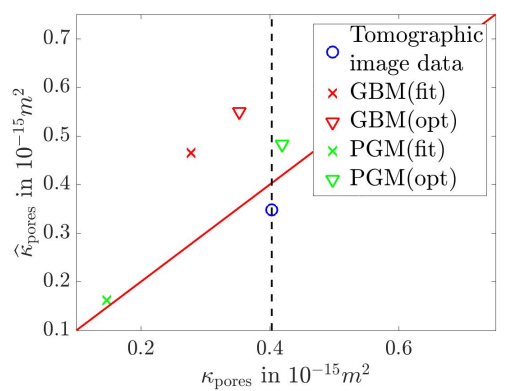

(c)

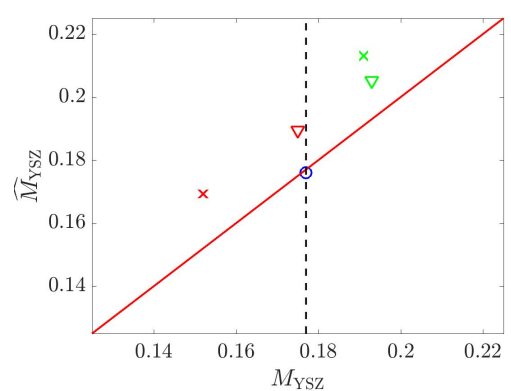

(b)

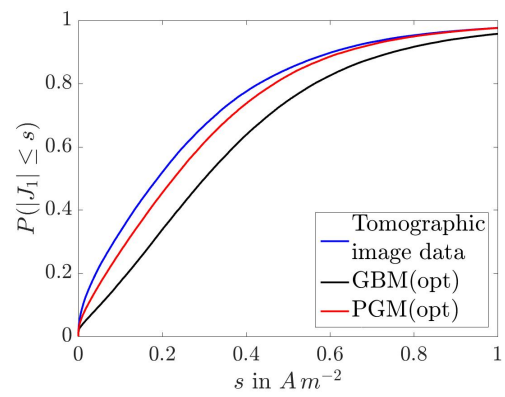

(d)

Fig. 5 Comparison of geometrically predicted values $\widehat{M}_{N i}, \widehat{M}_{\mathrm{YSZ}}$ and $\widehat{\kappa}_{\text {pores }}$ with numerically simulated values $M_{N i}, M_{\mathrm{YSZ}}$ and $\kappa_{\text {pores }}$ of effective transport properties. Results for permeability (c) in the pore space and for the $M$-factor of nickel (a) and YSZ (b). Additionally, the distribution function of the electrical current $J$ computed by means of numerical simulations within the nickel phase is shown for tomographic image and realizations of the microstructure models (d).

The predictions for the $M$-factor and effective permeability provided by the empirical formulas (18) and (19) are first compared to numerical FFT computa- 
tions in Fig. 5. For all models, the analytical predictions overestimate the effective properties in the average by $8 \%$ for YSZ and $17 \%$ for nickel. In effect, the difference is higher for the phase with lower volume fraction (nickel). For permeability, a significantly higher difference is observed, especially for the GBM model. Note however that, contrary to (18), the exponents in 19 have not been fitted to a database of virtual microstructure characteristics.

In the next step, we let model parameters vary to approach the three effective transport properties using virtual structures (see Table 3). Model GBM (fit) is in good agreement with the $M$-factors of solid phases, while permeability of the pore space is strongly underestimated. The reason for that is the good fit of structural characteristics of solid phases on the one hand, and the underestimation of constrictivity of the pore space $\beta_{3}$. When the microstructure characteristic $\beta_{3}$ is increased in the model, we obtain a more accurate prediction of $\kappa_{\text {pores }}$ (model GBM (opt)). However, the value of $\kappa_{\text {pores }}$ predicted by GBM(opt) is still significantly lower than the one computed for tomographic image data. The quality of the fit of PGM (fit) for $M_{\mathrm{Ni}}$ and $M_{\mathrm{YSZ}}$ is as good as the fit of GBM (fit). However, GBM (fit) underestimates $M_{\text {YsZ }}$ while PGM (fit) overestimates $M_{\text {YsZ }}$. The accordance of conductivities between model and tomographic image data does not change significantly when going from PGM (fit) to PGM (opt) and the corresponding microstructure characteristics $\varepsilon, \beta, \tau$ are close to each other. Still, a structural difference between PGM (fit) and PGM (opt) can be observed, which is reflected in the values of $r_{\max }$ and $r_{\min }$. In particular, a much better fit of $r_{\max }$ and $r_{\min }$ of the pore space is obtained in PGM (opt) compared to PGM (fit). As these structural characteristics influence permeability due to Equation (19), the better fit of $\kappa_{\text {pores }}$ in PGM (opt) compared to PGM (fit) is attributed to the better fit of $r_{\max }$ and $r_{\min }$. Concluding the comparison of effective transport properties, it turns out that the geometry-based estimation of model parameters for both the graph-based and the pluri-Gaussian model, presented in Abdallah et al. (2016) and Neumann et al. (2016), do not necessarily lead to the best fit regarding effective transport properties. This result indicates that the microstructure characteristics taken into account for the geometry-based estimation do not completely determine the effective transport properties. We discuss this effect in the following at the example of the $M$-factor of the nickel phase, which is overestimated by $20--30 \%$. Note however that this is the worst prediction of the three transport properties regarding the relative error. Nickel is also the phase with the lowest volume fraction. Presumably, the transport paths are the most tortuous for this phase, leading to regions that although connected to the percolating cluster, do not participate in the overall transport. We call this part the dead-end volume. The fraction of dead-end volume is not reflected in the notions of mean geodesic tortuosity and constrictivity and leads thus to a less accurate prediction of the $M$-factor with Equation (18), see Stenzel et al. (2017). It is not taken into account in the geometrical prediction of the $M$-factor as we are not aware of any rigorous definition of the dead-end volume purely based on geometry. Nevertheless, having computed the current in the nickel phase via numerical simulations as described in Section 4.1, its dead-end volume can be defined as the subset of the nickel phase where the current is approximately 0 . In order to give a better impression of the occurrence of dead-end volume, 2D visualizations are given in "Appendix B".

In Figure 5 the distribution function of the electrical current within the nickel phase, i.e., $F_{\left|J_{1}\right|}(s)=P\left(\left|J_{1}\right| \leq s\right)$, is shown for tomographic image data as well 
as for PGM(opt) and GBM(opt). The different increase in distribution functions at $s=0$ leads to a further underpinning of the assumption that the fraction of dead-end volume is different in the three microstructures. Compared to tomographic image data, the fraction of dead-end volume seems to be underestimated in PGM(opt) and GBM(opt), which would explain that the $M$-factor of the nickel phase is overestimated in the model realizations.

Overall, the joint fit of permeability and conductivities of the solid phases is not sufficiently good, if the parameters are estimated only on the basis of geometric characteristics. However, using the estimated model parameters as a starting point for a parameter estimation with respect to transport properties a much better fit can be obtained, at the price of a slightly less optimal fit of the geometric microstructure characteristics. Moreover, the fit of effective properties is better for the PGM compared to the GBM. In particular, the GBM underestimates permeability of the pore space.

\section{Comparison of Three-phase Microstructure models}

The considered microstructure models, one based on three random geometric graphs (GBM) and the other one based on two independent Gaussian random fields (PGM) are conceptually different from each other. By definition, connectivity properties of the three phases can be directly controlled by model parameters in the graph-based model. For a certain constellation of model parameters, it is even possible to ensure the complete connectivity of all three phases. Moreover, for a simplified version of the model $\left(b_{1}=b_{2}=b_{3}=2\right.$ and $\left.\gamma_{1}=\gamma_{2}=\gamma_{3}=1\right)$ results regarding the asymptotic behavior of the estimators for $\tau$ and $\beta$ have been obtained (Neumann et al., 2019). Complete connectivity of the phases cannot be achieved in the PGM for any parameter constellation by definition. Furthermore, the estimation of $\tau$ and $\beta$ has not yet been investigated for this model type from a theoretical point of view. However, the fit to tomographic image data, where the three phases exhibit good connectivity properties, shows that the connectivity can be sufficiently well reproduced by the PGM. To be precise, more than $99 \%$ of each phase is percolating in transport direction and thus contributes to flow or conduction processes. On the other hand, the relationships between model parameters and microstructure characteristics like volume fraction, specific surface area and twopoint coverage probability functions are well understood for the PGM (Lantuéjoul, 2013). Furthermore, in the present paper a formula for the expected length of the triple phase boundary per unit volume is derived. Such relationships are difficult to access analytically in the GBM and could not be derived so far. Thus a simulation study is performed to empirically relate model parameters with volume fractions of phases.

Using the relationships between model parameters and volume fractions as well as two-point coverage probability functions, the model parameters of the PGM can be directly estimated from image data. As volume fractions and two-point coverage probability functions can be estimated based on $2 \mathrm{D}$ images representing sections through a 3D microstructure, the model parameters of the PGM can also be estimated based on $2 \mathrm{D}$ image data assuming that the considered microstructure exhibits no anisotropy effects. This is a big advantage of the model as 3D imaging is much more expensive than $2 \mathrm{D}$ imaging. The model parameters in the GBM are 
numerically optimized regarding volume fractions, mean geodesic tortuosities and constrictivities of the solid phases as described in Neumann et al. (2016). This method cannot be used to estimate the model parameters based on 2D images, as the computation of $\tau$ and $\beta$ requires the full 3D information. Note that the numerical optimization is also more time-consuming compared to the estimation of model parameters in the pluri-Gaussian model.

The decision which of both models is preferable has to be made in dependence on the specific material or application for which the model is used. For the image data considered in the present paper, the PGM leads to a better fit regarding effective transport properties. Even if a better fit of constrictivity is obtained by the graph-based model, it underestimates permeability of the pore space and thus it does not allow to predict all relevant transport properties sufficiently well. So, for a model-based investigation on effective transport properties we suggest to prefer the PGM. However, due to the better fit of constrictivity, the GBM is-in the case of these particular structures-more appropriate for an investigation of the occurrence of bottlenecks. Besides that, in contrast to the PGM, the GBM allows for versatile modifications leading to topologically different microstructures. To be more precise, using different types of random geometric graphs, modeling the backbones of the three phases would enable promising extensions of the model toward higher flexibility.

\section{Conclusions}

Two three-phase 3D microstructure models, namely the pluri-Gaussian model of Abdallah et al. (2016), and the graph-based model of Neumann et al. (2016) are compared with respect to effective transport properties. The comparison is performed at an example of tomographic image data representing a three-phase microstructure occurring in SOFC anodes. For this purpose, both parametric models are fitted to image data based on geometrical characteristics of the microstructure. In a second step, the model parameters are optimized (using the fitted parameter constellations as starting points) with respect to permeability of the pore space as well as effective conductivities of the solid phases. By means of prediction formulas empirically derived in previous publications, we discuss the relationship between the fit of purely geometrical characteristics like volume fraction, mean geodesic tortuosity and constrictivity on the one hand and effective transport properties on the other hand. For the considered data set, the pluri-Gaussian model leads to a better fit of effective transport properties than the graph-based model, even if the pluri-Gaussian model overestimates the values of constrictivity. Finally, a comparison of the two microstructure models attempts to provide criteria in order to decide whether the pluri-Gaussian model or the graph-based model is used for a model-based investigation of microstructure-property relationships. Note that the presented models can also be used to study the microstructure influence on further effective properties like mechanical stress-strain curves in three-phase microstructures and are thus not restricted to effective conductivity and permeability. 


\section{Appendix}

\section{A Proof of Proposition 1}

To prove Proposition 1, we introduce a further stationary random set $\widetilde{\Xi}_{2}$ defined by $\widetilde{\Xi}_{2}=\{t \in$ $\left.\mathbb{R}^{3}: Y(t) \geq \lambda_{Y}\right\}$. Note that the specific surface areas $S_{1}$ and $\widetilde{S}_{2}$ of $\Xi_{1}$ and $\widetilde{\Xi}_{2^{-}}$respectivelycan be computed by

$$
S_{1}=\frac{2}{\pi} e^{-u_{Z}^{2} / 2} \sqrt{-\rho_{Z}^{\prime \prime}(0+)}
$$

and

$$
\widetilde{S}_{2}=\frac{2}{\pi} e^{-u_{Y}^{2} / 2} \sqrt{-\rho_{Y}^{\prime \prime}(0+)},
$$

if $Z$ and $Y$ are mean-square differentiable, see Equation (6.165) in Chiu et al. (2013) and Remark 7 in Ballani et al. (2012). In that case, $\rho_{Z}$ and $\rho_{Y}$ are twice differentiable from the right and $\rho_{Z}^{\prime \prime}(0+), \rho_{Y}^{\prime \prime}(0+)<0$, see Adler (1981). At first, we derive a formula which allows us to express $L_{\mathrm{TPB}}$ in terms of $S_{1}$ and $\widetilde{S}_{2}$. We show that

$$
L_{\mathrm{TPB}}=\pi S_{1} \widetilde{S}_{2} / 4 .
$$

Then, the assertion follows directly, when plugging Equations (20) and (21) into Equation (22). To prove Equation (22), note that the intersection $\Xi_{0}=\Xi_{1} \cap \Xi_{2} \cap \Xi_{3}$ is a motion-invariant random closed set and can be considered as a spatial fiber process in the sense of (Chiu et al., 2013, Section 8.4). Then, $\Xi_{0} \cap[0,1]^{2} \times\{o\}$ forms a motion-invariant point process with intensity $\vartheta_{0} \geq 0$. According to Equation (8.63) in Chiu et al. (2013) we obtain $L_{\mathrm{TPB}}=2 \vartheta_{0}$. Furthermore, note that the intersection of $\partial \Xi_{1}$ with an arbitrary one-dimensional subset of $\mathbb{R}^{3}$ forms a motion-invariant point process with intensity $\vartheta_{1} \geq 0$ satisfying $S_{1}=2 \vartheta_{1}$, see Equation(8.84) in (Chiu et al., 2013). In order to compute $\vartheta_{0}$, we use the independence of $Z$ and $Y$. This gives

$$
\begin{aligned}
\vartheta_{0} & =\mathbb{E} \mathcal{H}_{0}\left(\Xi_{0} \cap[0,1]^{2} \times\{o\}\right) \\
& =\int \mathbb{E}\left(\mathcal{H}_{0}\left(\partial \Xi_{1} \cap \partial \widetilde{\Xi}_{2} \cap\left([0,1]^{2} \times\{o\}\right)\right) \mid Y\right) \mathrm{d} P_{Y} \\
& =\vartheta_{1} \int \mathcal{H}_{1}\left(\partial \widetilde{\Xi}_{2} \cap\left([0,1]^{2} \times\{o\}\right) \mathrm{d} P_{Y}\right. \\
& =\frac{\pi}{8} S_{1} \widetilde{S}_{2},
\end{aligned}
$$

where the last equality is obtained by Equation(8.83) in Chiu et al. (2013).

\section{B Visualization of Dead-End Volume in 2D}

The dead-end volume turned out to serve as a reasonable interpretation of the difference observed between models (e.g. the PGM model) and the tomographic image data regarding the $M$-factor of the nickel phase. In order to visualize the dead-end volume, we have computed the current flow in a hypothetical structure. For this purpose, we considered YSZ and the pore space in a random 2D slice of tomographic image data and of realizations of the PGM model as conducting phases, while nickel is insulating. An electrical field $\left\langle E_{1}\right\rangle=1$ is applied for these $2 \mathrm{D}$ structures. This approach is useful for several reasons. First, it is easier to compare the field patterns between different structures in a purely-2D problem. This is however possible as long as discrepancies between the $M$-factors of model realizations and FIB-SEM images observed in $3 \mathrm{D}$ are also reflected in the results of $2 \mathrm{D}$ computations. Second, the nickel phase does not percolate in a $2 \mathrm{D}$ cut; hence we consider the flow in the complementary phase of the nickel (YSZ and pores). Indeed if the nickel phase of the models was representative of that observed in the FIB-SEM image, this would be the case also for its complementary. We observe a very significant difference between the two effective conductivities in $2 \mathrm{D}$, equal to $\sigma_{\mathrm{YSZ} \text {,pores }}=0.21$ for the realization of the PGM model and $\sigma_{\mathrm{YSZ} \text {, pores }}=0.31$ for the FIBSEM image. This suggests that the reason for the discrepancy is also present in the simpler 


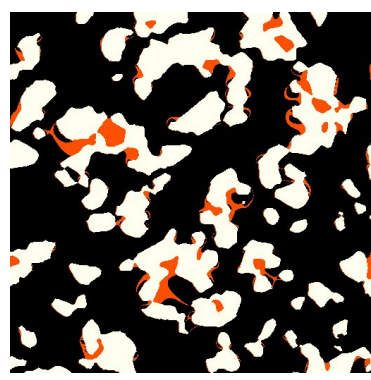

(a)

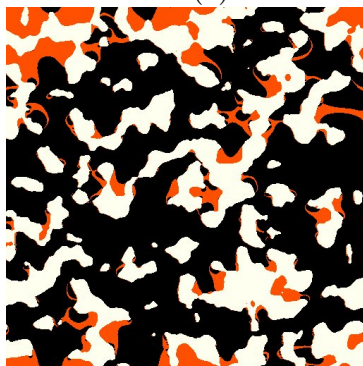

(c)

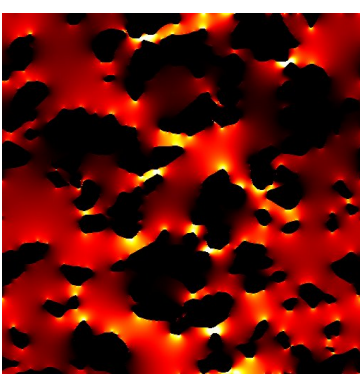

(b)

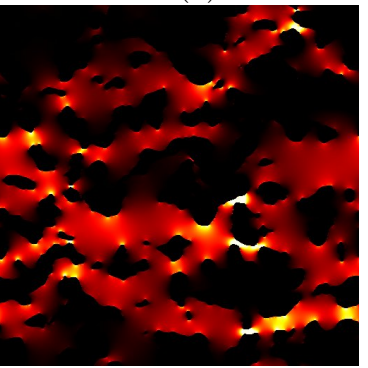

(d)

Fig. 6 Current field $J_{1}(\mathrm{~b}, \mathrm{~d})$ in $2 \mathrm{D}$ microstructures (a, c, flow occurs in the complement of the white phase) obtained as the union of the YSZ and pore phases: FIB-SEM image (a, b) and PGM model (c,d). The applied electrical field is $\left\langle E_{1}\right\rangle=1,\left\langle E_{2}\right\rangle=0$ and axis 1 is oriented left to right on the maps. The color maps $(b, d)$ indicate the current flow in the direction of axis 1 (lowest value in black, highest in white, values in-between in red and yellow). The color bar is restricted to current values between 0 and 2 . Zones of low current values $\left|J_{1}\right|<0.026$ are shown in red in maps (a) and (c).

2D problem. In Figure 6), regions of low current values $\left|J_{1}\right|<0.026$ are highlighted in red. These regions are considered as dead-end volume of the union of pores and YSZ. We observe that there is a significantly larger amount of such regions in realization of the PGM model than in tomographic image data. This can be related to much larger clusters in the model realization for the union of YSZ and of the pores than in tomographic image data (Figures 6a and $6 \mathrm{~b}$ ), which act as barriers. The presence of such barriers is consistent with a higher value of effective conductivity $\sigma_{\mathrm{YSZ} \text {,pores }}=0.31$ of tomographic image compared to the model realization $\left(\sigma_{\mathrm{YSZ}, \text { pores }}=0.21\right)$.

\section{Description of Computer Implementation of the Calculations}

In the following, we provide some technical details of the implementations used to simulate the virtual microstructures and their effective transport properties. The simulation of virtual microstructures by the GBM is implemented using Java in the framework of the software library Geostoch Mayer et al. (2004). Drawing one model realization with the parameters given in Table 1 takes about $25 \mathrm{~min}$ on a desktop computer. The code for generating virtual microstructures with the PGM is written in MATLAB MATLAB 2015b, The MathWorks (2015). One model realization with the parameters given in Table 2 takes about 5 min on a desktop computer. For the simulation of effective conductivity and permeability as described in Section 4.1, a Fortran code parallelized on a 24-cores machine is used. Calculations take about $2 \mathrm{~h}$ and $40 \mathrm{~min}$ for effective conductivity and permeability, respectively. 


\section{References}

Abdallah B, Willot F, Jeulin D (2015) Stokes flow through a Boolean model of spheres: representative volume element. Transport in Porous Media 109(3):711-726

Abdallah B, Willot F, Jeulin D (2016) Morphological modelling of three-phase microstructures of anode layers using SEM images. Journal of Microscopy 263(1):51-63

Adler RJ (1981) The Geometry of Random Fields. J. Wiley \& Sons, Chichester

Ballani F, Kabluchko Z, Schlather M (2012) Random marked sets. Advances in Applied Probability 44(3):603-616

Bertei A, Chueh CC, Pharoah JG, Nicolella C (2014) Modified collective rearrangement sphereassembly algorithm for random packings of nonspherical particles: Towards engineering applications. Powder Technology 253:311-324

Brabec CJ, Heeney M, McCulloch I, Nelson J (2011) Influence of blend microstructure on bulk heterojunction organic photovoltaic performance. Chemical Society Reviews 40(3):11851199

Cai Q, Adjiman CS, Brandon NP (2011) Modelling the 3D microstructure and performance of solid oxide fuel cell electrodes: Computational parameters. Electrochimica Acta 56(16):58045814

Chiu SN, Stoyan D, Kendall WS, Mecke J (2013) Stochastic Geometry and its Applications, 3rd edn. J. Wiley \& Sons, Chichester

Hirsch C, Neuhäuser D, Schmidt V (2013) Connectivity of random geometric graphs related to minimal spanning forests. Advances in Applied Probability 45(1):20-36

Holzer L, Wiedenmann D, Münch B, Keller L, Prestat M, Gasser P, Robertson I, Grobéty B (2013) The influence of constrictivity on the effective transport properties of porous layers in electrolysis and fuel cells. Journal of Materials Science 48:2934-2952

Holzer L, Pecho O, Schumacher J, Marmet P, Stenzel O, Büchi FN, Lamibrac A, Münch B (2017) Microstructure-property relationships in a gas diffusion layer (GDL) for polymer electrolyte fuel cells, part I: Effect of compression and anisotropy of dry GDL. Electrochimica Acta 227:419-434

Kanit T, Forest S, Galliet I, Mounoury V, Jeulin D (2003) Determination of the size of the representative volume element for random composites: statistical and numerical approach. International Journal of Solids and Structures 40:3647-3679

Kenney B, Valdmanis M, Baker C, Pharoah JG, Karan K (2009) Computation of tpb length, surface area and pore size from numerical reconstruction of composite solid oxide fuel cell electrodes. Journal of Power Sources 189(2):1051-1059

Kirkpatrick DR, Radke JD (1985) A framework for computational morphology. In: Toussaint GT (ed) Computational Geometry, North-Holland, Amsterdam, pp 217-248

Lantuéjoul C (2013) Geostatistical Simulation: Models and Algorithms. Springer, Berlin

Matheron G (1975) Random Sets and Integral Geometry. J. Wiley \& Sons, New York

MATLAB 2015b, The MathWorks (2015) www.matlab.com

Mayer J, Schmidt V, Schweiggert F (2004) A unified simulation framework for spatial stochastic models. Simulation Modelling Practice and Theory 12(5):307-326

Molchanov I (1997) Statistics of the Boolean Model for Practitioners and Mathematicians. J. Wiley \& Sons, Chichester

Møller J, Waagepetersen RP (2004) Statistical Inference and Simulation for Spatial Point Processes. Chapman \& Hall/CRC, Boca Raton

Moulinec H, Suquet P (1994) A fast numerical method for computing the linear and non linear mechanical properties of the composites. Comptes Rendus de l'Académie des Sciences Série II 318:1417-1423

Moussaoui H, Laurencin J, Gavet Y, Delette G, Hubert M, Cloetens P, Le Bihan T, Debayle $\mathrm{J}$ (2018) Stochastic geometrical modeling of solid oxide cells electrodes validated on 3D reconstructions. Computational Materials Science 143:262-276

Nelder JA, Mead R (1965) A simplex method for function minimization. The Computer Journal $7: 308-313$

Neumann M, Staněk J, Pecho OM, Holzer L, Beneš V, Schmidt V (2016) Stochastic 3D modeling of complex three-phase microstructures in SOFC-electrodes with completely connected phases. Computational Materials Science 118:353-364

Neumann M, Hirsch C, Staněk J, Beneš V, Schmidt V (2019) Estimation of geodesic tortuosity and constrictivity in stationary random closed sets. Scandinavian Journal of Statistics In print, doi:10.1111/sjos.12375. 
Ohser J, Schladitz K (2009) 3D Images of Materials Structures: Processing and Analysis. J. Wiley \& Sons, Weinheim

Pecho OM, Stenzel O, Gasser P, Neumann M, Schmidt V, Hocker T, Flatt RJ, Holzer L (2015) 3D microstructure effects in Ni-YSZ anodes: Prediction of effective transport properties and optimization of redox-stability. Materials 8(9):5554-5585

Prakash BS, Kumar SS, Aruna ST (2014) Properties and development of Ni/YSZ as an anode material in solid oxide fuel cell: A review. Renewable and Sustainable Energy Reviews $36: 149-179$

Scholz C, Wirner F, Klatt MA, Hirneise D, Schröder-Turk GE, Mecke K, Bechinger C (2015) Direct relations between morphology and transport in Boolean models. Physical Review E 92(4):043023

Stenzel O, Pecho OM, Holzer L, Neumann M, Schmidt V (2016) Predicting effective conductivities based on geometric microstructure characteristics. AIChE Journal 62:1834-1843

Stenzel O, Neumann M, Pecho OM, Holzer L, Schmidt V (2017) Big data for microstructureproperty relationships: A case study of predicting effective conductivities. AIChE Journal 63(9):4224-4232

Suzuki T, Hasan Z, Funahashi Y, Yamaguchi T, Fujishiro Y, Awano M (2009) Impact of anode microstructure on solid oxide fuel cells. Science 325(5942):852-855

Tjaden B, Brett DJL, Shearing PR (2018) Tortuosity in electrochemical devices: a review of calculation approaches. International Materials Reviews 63(2):47-67

Torquato S (2013) Random Heterogeneous Materials: Microstructure and Macroscopic Properties. Springer, New York

Westhoff D, Van Franeker JJ, Brereton T, Kroese DP, Janssen RAJ, Schmidt V (2015) Stochastic modeling and predictive simulations for the microstructure of organic semiconductor films processed with different spin coating velocities. Modelling and Simulation in Materials Science and Engineering 23(4):045003

Wiegmann A (2007) Computation of the permeability of porous materials from their microstructure by FFF-Stokes. http://kluedo.ub.uni-kl.de/files/1984/bericht129.pdf, accessed 22 July 2015

Willot F, Abdallah B, Pellegrini YP (2014) Fourier-based schemes with modified green operator for computing the electrical response of heterogeneous media with accurate local fields. International Journal of Numerical Methods in Engineering 98:518-533 